\title{
Public Health and Medicine
}

\section{At the Crossroads}

By HERMAN E. HILlebOE, M.D., M.P.H.

In these days of disorder, change, and impending disaster, we who are dedicated to the health and welfare of the people face tasks of greater challenge than we have ever known before. We have arrived at a stage in our social development, whether desirable or not, at which the people turn to us increasingly for aid and guidance. This is a grave responsibility. Under its burden and facing its humane demands, we are compelled to review the adequacy of our guiding principles.

Public health is at the crossroads. We must now choose the direction that we shall take in the future, or it will be chosen for us.

The concept of health department services to the people has undergone dramatic change. Advancing medical knowledge and public health activities have sharply decreased morbidity and mortality from the communicable diseases. Improved working conditions, better nutrition, more attention to preventive medical services in the schools, and similar social forces have contributed to improved health and welfare. Environmental sanitation programs are well on their way. Means have been provided and successful methods have been developed for the expansion of environmental health activities.

It is plain that, in the future, the theory and practice of public health must be expanded to

Dr. Hilleboe, commissioner of health for the State of New York, presented this paper at the first general session of the forty-eighth annual health conference of the State of New York, at Lake Placid, June 4, 1952. include a vigorous attack upon the chronic diseases, with special attention to heart disease and cancer, which are the principal killers and disablers in our Nation.

We need new knowledge, new methods, and new measurements for evaluating our programs. As the demands of communicable disease control continue to decrease, we must utilize our acquired skills in combating the ravages of the chronic diseases, an increasingly crucial problem for the private practitioner and the public health physician.

\section{Death Rates: New York}

Where do we stand in New York State? The 1951 death rate from all causes in the State was 10.5 per 100,000 population. The death rate has never been lower. In 1940 the rate was 11.1. The degree of improvement is greater than these figures indicate. In the last decade the proportion of older persons, among whom mortality is inevitably high, has increased. Had the age distribution of the population in 1951 been the same as in 1940, the death rate would have been 9.2 instead of 10.5.

Infant mortality, 23.7 per 1,000 live births, was the lowest ever experienced and represents a drop of 36 percent since 1940 . In the same interval, neonatal mortality decreased 28 percent, while that between 1 month and 1 year dropped 55 percent. Maternal mortality, 4.9 per 10,000 live and still births, also dropped to a new low record and was less than one-fifth of the rate in 1940.

New low levels were reached in 1951 by deaths from streptococcal sore throat (including 
scarlet fever), whooping cough, and diphtheria. Deaths from streptococcal sore throat numbered 21 as contrasted with 107 in 1940. Deaths from whooping cough numbered 11, while in 1940 there were 138 . Only two deaths were attributed to diphtheria; the previous low mark of nine occurred in 1944.

Among the diseases to which all ages are subject, the death rate per 100,000 population for 1951 in New York State, including New York City, was 4.0 for syphilis and 23.5 for tuberculosis. The death rates for these two human scourges were lower than ever before in our history. Indeed, the tuberculosis death rate for 1951 showed a drop of 9 percent over that of the preceding year and was 50 percent less than the rate in 1940 . In this 11-year period, the tuberculosis death rate in New York City dropped 46 percent-from 54.4 to 29.5 . In the rest of the State, the reduction was 50 percentfrom 33.7 to the 1951 rate of 16.7 .

Contrast these low rates with the awesome death rates of 184.4 from cancer and 459.8 from heart disease. To state them another way: 43 of every 100 deaths in upstate New York were due to heart disease; 17 out of every 100 deaths were due to cancer; and 11 out of every 100 deaths were from intercranial lesions of vascular origin. It is estimated that 1 out of every 20 persons in the United States suffers from some disorder of the cardiovascular system; every other death after the age of 45 years is caused by heart disease.

\section{Cardiovascular Diseases}

Public health epidemiology has made distinct contributions in the field of the cardiovascular diseases. The causes of beriberi, scurvy, and pellagra were elucidated almost solely by epidemiological methods-these methods contributed much to the understanding and prevention of heart disease associated with nutritional deficiencies. Again, the aggregation of rheumatic fever in families and the probable importance of hemolytic streptococcal infections in precipitating heart attacks were discovered largely by epidemiological studies.

In spite of these memorable advances, we seriously lack epidemiological knowledge of many types of cardiovasoular disease. Our in- formation concerning the prevalence of cardiac ailments is limited to selected fractions of the population. The causes of atherosclerosis, hypertension, and rheumatic fever are still unknown. Epidemiologists would be greatly aided in their study of the etiology and demography of cardiovascular diseases if public health workers could devise and test mass methods of screening the adult population for these diseases. Their use in large population groups would give us valuable data on the extent of the problem, and would enable us to bring large numbers of early cases under the supervision of family physicians.

\section{Secondary Preventive Services}

Research is being intensified on a broad front to find preventive measures that will help people to avoid heart disease. While this type of fundamental research is being pursued, we can use other approaches to the control of heart disease by strengthening our secondary preventive services. These are directed at the earliest detection of asymptomatic heart disease and the detection of early disease, so that the progression of clinical symptoms may be halted and further damage to the cardiovascular system avoided or minimized.

It is in this field of secondary prevention that health departments can make significant contributions. It is not enough to offer postgraduate courses for physicians, to develop nutritional weight control programs for the obese, or to acquaint the public with the problems of heart disease by means of the techniques of health education. If we are to assault the chronic diseases with the same vigor and success with which we attacked the communicable diseases, we must employ epidemiological methods in the exploration of these new fields. With only a few minor exceptions, we don't know how much heart disease there is in our communities. We do not know what types of heart disease are prelavent; nor do we know what types can be benefited by early detection. For atherosclerosis and coronary disease, we have no accurate mass case-finding methods.

Where, then, do we start and what do we do? There are two approaches that can be used by any health department large enough to employ 
full-time public health physicians and with courage enough to explore uncharted fields in public health.

Athero-coronary disease is the major contributor to death in the cardiovascular group. The various tests used to detect it early have not been evaluated either as to their relative efficiency or mass applicability. However, such studies can be undertaken by any large health department that can obtain random samples of the adult population at risk, and that has both the equipment needed for a battery of tests and the necessary professional and technical staff to perform and evaluate the tests.

This is a formidable task, but so was the early detection of syphilis and tuberculosis. It is better to spend the next few years in the determination of the reliability, validity, yield, cost, and acceptability of screening methods for coronary disease than to wait and muddle through with whatever might be available in the distant future.

Public demands for heart disease control programs are daily gaining momentum. It is inevitable that we will find new screening methods as we test the old, discard the inefficient, and develop those that single out most efficiently the potential and real, though obscure, cases.

Since early this year the New York State Health Department has had a small study group working on this approach to the problems of coronary disease and hypertension. We will launch our program on a group of several hundred key personnel in our State government in the age groups over 40, in which coronary disease and hypertension death rates are high.

We intend to seek out the most effective methods of detecting coronary insufficiency before occlusion or hypertension or cardiac hypertrophy occur. As soon as we achieve adequate information concerning mass screening methods and have accurate knowledge of the groups most at risk, we shall extend our activities to a full-scale community program, with the close cooperation of health departments, private practitioners, and medical schools.

\section{Public Health Approach}

Those of us over 40 years of age have a personal as well as a professional interest in the public health approach to cardiovascular disease. Blumgart (1) found that among several hundred persons over 40 who died of noncardiac causes and who had shown no evidence of heart disease, 40 percent exhibited on autopsy considerable hardening and narrowing of the coronary arteries. I urge initiation and pursuit of specific epidemiological studies.

The second approach to the problem of heart disease can best be made by county and city health departments because of their proximity to the sufferers from heart disease who will be needed for such studies. Specifically, I refer to the several hundred thousand chest $\mathrm{X}$-ray films taken each year for the purpose of tuberculosis case finding. Rutstein, Williamson, and Moore (2), in their Boston study which was reported in November 1951, have clearly demonstrated that it is possible by means of $70-\mathrm{mm}$. photofluorographic film to identify in the general population a significant number of persons who have previously unknown heart disease. To be sure, skilled cardiologists must reread the films. When this is done, three times as many abnormal hearts are found as when lessskilled film readers review the films. In addition, persons with abnormal hearts must be referred to clinicians and followed up by their private physicians.

Here is an extraordinary opportunity for the health officer to initiate cooperative activity of major importance between the health department and the medical profession. Great benefits will accrue to thousands of cardiac patients in terms of productivity, personal happiness, and prolonged life.

Rutstein and his colleagues (2) point out that all newly discovered cases of rheumatic fever were benefited, because they learned to avoid further exposure to streptococcal infection and subacute bacterial endocarditis after operation or tooth extraction. In addition, many heart patients were more amenable to the techniques of vocational rehabilitation. Similarly, newly discovered cases of hypertensive or arteriosclerotic heart disease with auricular fibrillation, angina pectoris, and marked obesity certainly could be benefited by prompt referral to medical care. There was also a miscellaneous group of cases-congenital syphil- 
itic, thyrotoxic heart disease, and pericardial tumors-that were greatly improved by medical or surgical therapy.

\section{Private Physician Benefited}

Such case finding through mass surveys of well people will enable the private physician to see patients in the asymptomatic stages of their disease, when therapy has its greatest effectiveness. Moreover, public health activities in this field will supplement the practicing physician's case finding in his office. This is just one example of the kind of epidemiological work local health departments can conduct successfully.

\section{Priority for Chronic Diseases}

The impersonal numbers on the mortality tables cannot convey the tragic stories of human suffering and family disruption that follow in the wake of crippling attacks of heart disease. The magnitude of the problem gives high priority, on the agenda of the health officer, to cardiovascular disease study and control.

Similar epidemiological studies should be undertaken in the field of cancer. Such an enterprise will also demand the active cooperation of the practicing physician and the public health worker.

In attempting to conquer heart disease, cancer, and the other chronic diseases, it is essential that we enlarge the scope of coverage and the number of services which the medical profession and public health agencies can provide. In New York State, we have been for years keenly aware of the necessity of providing medical care for the indigent, and we can boast of the best medical care program for welfare recipients of any State in the Nation.

To make medical care for the indigent even more certain and universal, the New York State departments of welfare and health recently took a significant step forward in cooperative governmental endeavor. The State health department established the new position of assistant commissioner of health (for welfare). One of our key public health administrators was assigned to the Department of Social Welfare to develop and evaluate the medical care program for recipients of public assistance. It is a unique arrangement in government. The new assistant commissioner is responsible to the commissioner of health for professional guidance and has full access to all the information and facilities of the State health department; administratively, he is responsible to the commissioner of social welfare in whose department his office is located.

These medical care programs for more than 3 million persons are locally administered and use the facilities, services, and personnel of both the public and voluntary medical agencies and institutions. Through local hospitals and dispensaries, approximately 850,000 persons receive medical care annually at a cost of $\$ 106$,000,000 . Through local public welfare agencies, 250,000 needy sick persons are cared for at home and in doctors' offices at a yearly expenditure of more than $\$ 6,000,000$ in public assistance funds. We have an unequaled opportunity to integrate preventive services of health departments into curative services of welfare departments.

Through this interdepartmental cooperation, private physicians, public health and welfare officials, nurses, social workers, rehabilitation specialists, and many others will be working together in an integrated, sustained effort to restore to health and to useful community roles thousands of the diseased and disabled whose impairments have reduced them to economic and social dependency.

It is by means of such cooperative enterprise that we may make a beginning toward the achievement of our high goals. By such realistic teamwork we can conserve efforts, personnel, and funds, and we can measure and surmount the barriers which daily we encounter in our work.

\section{Philosophical Merger Necessary}

Public health agencies cannot do these gigantic tasks alone. It will require the cooperative efforts of the whole of society. Particularly will it require a philosophical merger of the policies of medical societies and public health agencies. Clinical medicine is becoming increasingly aware of its role as a social instrument, and public health practice is perceiving 
more keenly the problems of the individual in his relation to the family and the community in which he lives and works. But we need a closer working arrangement before we can be effective in controlling heart disease and cancer.

Lest it be feared that by working closely with official public health agencies the medical profession will suffer a loss of freedom, it should be said that complete freedom is not necessarily the natural state of human beings in a crowded society. It is something that can be obtained only within natural limits by continually clearing away obstructions. In seeking freedom in a medical field or profession, it is not enough to say that we want special privilege and the license to do what we like. We must first answer the question, "Freedom for what?"

What we seek is freedom to serve. To be sure, the practicing physician should have every freedom in the exercise of his judgment in the care of his patients. Much of the value of medicine rests on the willingness of sincere physicians to spend unlimited time and effort because they feel responsible for the patient's life and happiness. When this personal feeling is artificial or absent, the work of physicians descends to a low level of effectiveness.

We ought to strive for and insist upon two things that can be coexistent-professional freedom and professional responsibility. Properly viewed, the work of the whole medical profession, which includes public health, is not done in our private interests but in the interest of the community.

With this broad view of professional freedom and responsibility, the private physician and the public health physician can serve the individual and the community to best advantage by working together. They must not be driven by a desire for personal gain alone, but by a willingness to serve the common good. The breadth of this concept emphasizes the scope of action in which all members of the medical profession should participate. Without such a wide horizon, we shall become ingrown and isolated from the dynamic trends of social evolution.

If we are to achieve this common objective of professional cooperation, it is vital that the medical profession, which holds a key position in the social order, close its ranks and that public health and private physicians contribute their full quota to the common effort. We must be prepared, while retaining the best of our traditions, to adjust our efforts to the changing conditions in society.

Medicine changes with time and place, and we must keep abreast of these changes. This will require mutual understanding and faith and trust. The result will be a fuller appreciation on the part of the public health worker of the problems of the general practitioner, plus enlightened awareness on the part of the clinical physician of the responsibilities and special attributes of the public health physician.

We stand at the crossroads. We must take new direction and answer the medical and social questions posed by the chronic diseases as we continue to carry the burdens of our regular work. With courage and with faith in the creative mind of man, we shall succeed.

\section{REFERENCES}

(1) Blumgart, Hermann L.: Coronary disease : Clinical-pathological correlations and physiology. Bull. New York Acad. Med. 27: 693-710 (1951).

(2) Rutstein, David D., Williamson, C. R., and Moore, F. E. : Heart disease case finding by means of 70-millimeter photofluorographic films. Group I. Circulation 4: 641-651 (1951). 